STRUCTURAL BIOLOGY COMMUNICATIONS

ISSN 2053-230X

Received 8 January 2019

Accepted 13 March 2019

Edited by I. Tanaka, Hokkaido University, Japan

₹ These authors contributed equally to this work.

$\S$ Present address: Faculty of Pharmacy,

Keio University, 1-5-30 Shibakoen, Minato-ku, Tokyo 105-8512, Japan.

Keywords: peptide transporter; major facilitator superfamily; lipidic cubic phase; single-particle cryo-electron microscopy; membrane transporter; oligomerization; cryo-EM; X-ray crystallography.

PDB references: PepT $_{\mathrm{So} 2}$, cryo-EM structure, 6ji1; X-ray structures, 6jkc; 6jkd

Supporting information: this article has supporting information at journals.iucr.org/f

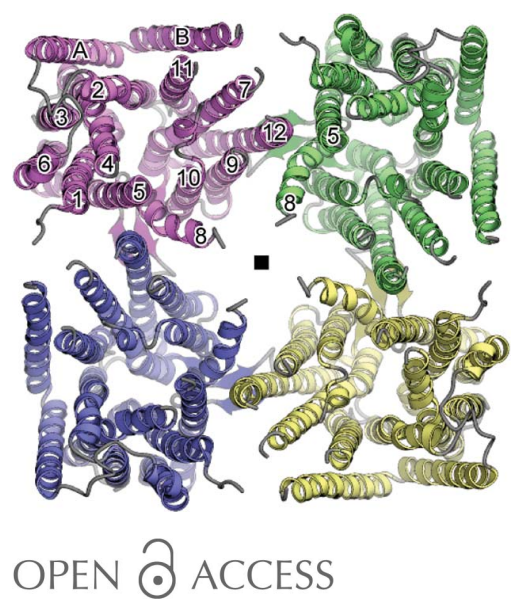

\section{Structural basis for oligomerization of the prokaryotic peptide transporter $\mathrm{PepT}_{\mathrm{So} 2}$}

\author{
Reina Nagamura, ${ }^{\mathrm{a}} \neq$ Masahiro Fukuda, ${ }^{\mathrm{a}} \neq \S$ Akihiro Kawamoto, ${ }^{\mathrm{b}}$ Kyoko Matoba, \\ Naoshi Dohmae, ${ }^{\mathrm{c}}$ Ryuichiro Ishitani, ${ }^{a}$ Junichi Takagi ${ }^{\mathrm{b}}$ and Osamu Nureki ${ }^{\mathrm{a} *}$
}

\begin{abstract}
${ }^{a}$ Department of Biological Sciences, Graduate School of Science, The University of Tokyo, 7-3-1 Hongo, Bunkyo-ku, Tokyo 113-0033, Japan, ' Institute for Protein Research, Osaka University, 3-2 Yamadaoka, Suita, Osaka 565-0871, Japan, and ${ }^{\mathrm{C}}$ RIKEN Advanced Science Institute, 2-1 Hirosawa, Wako-shi, Saitama 351-0198, Japan. *Correspondence e-mail: nureki@bs.s.u-tokyo.ac.jp
\end{abstract}

Proton-dependent oligopeptide transporters (POTs) belong to the major facilitator superfamily (MFS) and transport dipeptides and tripeptides from the extracellular environment into the target cell. The human POTs PepT1 and PepT2 are also involved in the absorption of various orally ingested drugs. Previously reported structures revealed that the bacterial POTs possess 14 helices, of which $\mathrm{H} 1-\mathrm{H} 6$ and $\mathrm{H} 7-\mathrm{H} 12$ constitute the typical MFS fold and the residual two helices are involved in the cytoplasmic linker. PepT $\mathrm{S}_{\mathrm{So} 2}$ from Shewanella oneidensis is a unique POT which reportedly assembles as a $200 \mathrm{kDa}$ tetramer. Although the previously reported structures suggested the importance of $\mathrm{H} 12$ for tetramer formation, the structural basis for the $\mathrm{PepT}_{\mathrm{So}_{2} \text {-specific }}$ oligomerization remains unclear owing to the lack of a high-resolution tetrameric structure. In this study, the expression and purification conditions for tetrameric PepT $\mathrm{T}_{\mathrm{So} 2}$ were optimized. A single-particle cryo-EM analysis revealed the tetrameric structure of $\mathrm{PepT}_{\mathrm{So} 2}$ incorporated into Salipro nanoparticles at $4.1 \AA$ resolution. Furthermore, a combination of lipidic cubic phase (LCP) crystallization and an automated data-processing system for multiple microcrystals enabled crystal structures of $\mathrm{PepT}_{\mathrm{So} 2}$ to be determined at resolutions of 3.5 and $3.9 \AA$. The present structures in a lipid bilayer revealed the detailed mechanism for the tetrameric assembly of $\mathrm{PepT}_{\mathrm{So} 2}$, in which a characteristic extracellular loop (ECL) interacts with two asparagine residues on H12 which were reported to be important for tetramerization and plays an essential role in oligomeric assembly. This study provides valuable insights into the oligomerization mechanism of this MFS-type transporter, which will further pave the way for understanding other oligomeric membrane proteins.

\section{Introduction and research aim}

Proton-dependent oligopeptide transporters (POTs) belong to the major facilitator superfamily (MFS) and transport shortchain peptides from the extracellular environment into the target cell. The human POTs PepT1 and PepT2 are involved in the absorption of digested peptides and various orally ingested drugs, including antibiotics, antivirals and anticancer agents. Previously reported structures revealed that the bacterial POTs possess 14 helices, of which $\mathrm{H} 1-\mathrm{H} 6$ and $\mathrm{H} 7-\mathrm{H} 12$ constitute the typical MFS fold and the residual two helices, $\mathrm{HA}$ and $\mathrm{HB}$, are inserted between $\mathrm{H} 6$ and $\mathrm{H} 7$ (Doki et al., 2013; Guettou et al., 2013; Newstead et al., 2011; Parker et al., 2017; Zhao et al., 2014). PepT So2 $_{\text {from Shewanella oneidensis is }}$ a unique POT transporter that has been reported to assemble as a $200 \mathrm{kDa}$ tetramer in the detergent-solubilized form (Guettou et al., 2013). Two crystal structures of PepT $\mathrm{So2}_{\mathrm{S} 2}$ have been reported: tetrameric and dimeric structures determined 
at resolutions of 4.6 and $3.2 \AA$, respectively (Guettou et al., 2013). Although the resolution of the dimeric structure was much higher, the dimer interface was stabilized by a zinc ion contained in the crystallization conditions, suggesting that this dimeric form represents a crystal-packing artifact. Recently, a cryo-electron microscopy (cryo-EM) structure of the PepT $\mathrm{S}_{\mathrm{So} 2}$ tetramer embedded in Salipro nanoparticles was reported at $6.5 \AA$ resolution (Frauenfeld et al., 2016). A structural comparison of the dimeric and tetrameric $\mathrm{PepT}_{\mathrm{So} 2}$ structures suggested that subtle structural changes of $\mathrm{H} 12$ and the unconserved Asn468 on $\mathrm{H} 12$ are important for tetramer formation (Guettou et al., 2013). However, the structural basis for the $\mathrm{PepT}_{\mathrm{So} 2}$-specific oligomerization remained unclear owing to the insufficient resolution of the tetrameric structure.

In this study, we overexpressed PepT $\mathrm{T}_{\mathrm{So} 2}$ in Escherichia coli and optimized the expression conditions. During the expression trials, we found that the number of histidine tags fused to the C-terminus drastically affected the oligomerization state of PepT $\mathrm{So2}_{\mathrm{So}}$ Tetramer formation was confirmed by a singleparticle analysis using cryo-EM and was further analyzed by $\mathrm{X}$-ray crystallographic analyses using lipidic cubic phase (LCP) crystallization (Caffrey \& Cherezov, 2009). As a result of the crystallization trials, microcrystals were obtained under multiple conditions. We finally determined structures of $\mathrm{PepT}_{\mathrm{So2} 2}$ from two different crystal forms at 3.5 and $3.9 \AA$ resolution by merging small wedge data sets from multiple microcrystals (Yamashita et al., 2018).

$\mathrm{PepT}_{\mathrm{So} 2}$ forms a tetramer in both of the crystal forms, and the structures superimpose well (with an r.m.s.d. of $0.83 \AA$ for all $\mathrm{C}^{\alpha}$ atoms). A slight movement of $\mathrm{H} 12$ was also observed, as in the previous structure, suggesting the importance of $\mathrm{H} 12$ for tetramer formation. Furthermore, the tetrameric assembly appears to be stabilized by hydrogen bonds between the asparagine residues in $\mathrm{H} 12$ and the unconserved extracellular loop (ECL), which was not well resolved in the previously determined low-resolution tetrameric structures (Frauenfeld et al., 2016; Guettou et al., 2013). Although the physiological significance of the tetrameric assembly of $\mathrm{PepT}_{\mathrm{So} 2}$ remains unclear, the oligomerization is possibly essential for the stable expression of $\mathrm{PepT}_{\mathrm{So} 2}$ in the plasma membrane. This study provides valuable insights into the oligomerization mechanism of this MFS-type transporter, which will further facilitate the understanding of other oligomeric membrane proteins.

\section{Materials and methods}

\subsection{Macromolecule production}

The gene encoding full-length Pep $\mathrm{T}_{\text {So2 }}$ (UniProt Q8EHE6) was subcloned into a pET-modified vector (Nishizawa et al., 2013) which contains the DNA sequence encoding the Tobacco etch virus (TEV) protease cleavage site (ENLYFQG) followed by a $\mathrm{His}_{6}$ or $\mathrm{His}_{8}$ tag at the 3'-terminus. The macromolecule-production information is summarized in

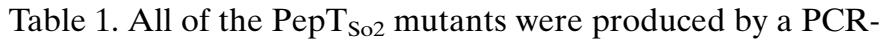
based site-directed mutagenesis method using PrimeSTAR Max DNA polymerase (Takara).
Table 1

Macromolecule-production information.

\begin{tabular}{|c|c|}
\hline Source organism & S. oneidensis \\
\hline DNA source & UniProt Q8EHE6 \\
\hline Expression vector & Modified pET-28a \\
\hline Expression host & E. coli Rosetta 2 (DE3) \\
\hline $\begin{array}{c}\text { Complete amino-acid sequence } \\
\text { of the construct produced } \dagger\end{array}$ & $\begin{array}{l}\text { MTLGTNQVSKTHSFMTVSLIELWERFGYYG } \\
\text { MQALIVYFMVQRLGFDDSRANLVWSACA } \\
\text { ALIYVSPAIGGWVGKILGTKRTMLLGA } \\
\text { GILSVGYLMTVPTENTWFMFSALGVIV } \\
\text { VGNGLFKPNAGNLVRKIYEGDDSKIDSA } \\
\text { FTIYYMAVNVGSTFSMLLTPWIKDYVNA } \\
\text { QYGNEFGWHAAFAVCCVGILVGLGNYAL } \\
\text { MHKSLANYGSEPDTRPVNKKLAIVLAL } \\
\text { AALSVVASAIILEYEDVARVFVYAAGVA } \\
\text { VLGIFFHLIRTSEPSERAGLIAALILTV } \\
\text { QTVFFFIFYQQMSTSLALFALNVDWF } \\
\text { QVFGTHLWTWSPAQFQALNPIWIMVLSP } \\
\text { VLAWSYSWAGRNNKDFSIAAKFALGFAV } \\
\text { VAIGFFIYGFAGQFAVNGKTSSWVMIWG } \\
\text { YASYSLGELLVGLGLAMIARYVPARMG } \\
\text { GFMMGAYFASGISQYLGGVVANFASVP } \\
\text { QDLVDPLQTLPVYTNLFNKLGVAAVVCT } \\
\text { IIALAVLPLMRRLTESHHAHSSIENNAA } \\
\text { ASLRDVKAEQLESSGENLYFQ (GQFTSS } \\
\text { VHHHHHH) }\end{array}$ \\
\hline
\end{tabular}

$\dagger$ The cloning artifacts are underlined. The residues cleaved by TEV protease are in parentheses.

\subsection{Optimization of the expression conditions for $\boldsymbol{P e p}_{\mathrm{So} 2}$}

The plasmid was introduced into Escherichia coli Rosetta 2 (DE3) cells (Novagen) or genetically modified C41 (DE3) cells harboring the pRARE plasmids encoding the tRNAs for codons that are rarely used in E. coli. The transformed cells were grown in $2.5 \mathrm{ml}$ Luria-Bertani (LB) medium containing $50 \mu \mathrm{g} \mathrm{ml}^{-1}$ ampicillin with or without $0.4 \%$ glucose or in $2.5 \mathrm{ml}$ Terrific Broth (TB) at $310 \mathrm{~K}$. When the absorbance at $600 \mathrm{~nm}\left(A_{600}\right)$ reached $0.5-0.8$, protein expression was induced with $0.5 \mathrm{~m} M$ isopropyl $\beta$-D-1-thiogalactopyranoside (IPTG) and the cells were grown for about $18 \mathrm{~h}$ at $293 \mathrm{~K}$. The cells were centrifuged at $8000 \mathrm{~g}$ for $1 \mathrm{~min}$ and were then suspended in solubilization buffer $[50 \mathrm{~m} M$ Tris- $\mathrm{HCl} \mathrm{pH} 8.0,300 \mathrm{~m} M \mathrm{NaCl}, 15 \mathrm{~m} M$ imidazole, $0.5 \mathrm{~m} M$ Tris(2-carboxyethyl)phosphine (TCEP), 10\% glycerol, 2\% $n$-dodecyl- $\beta$-D-maltoside (DDM)] supplemented with cOmplete EDTA-free Protease Inhibitor Cocktail (Roche). The cells were disrupted by sonication using a Bioruptor UCW-310 (Cosmo Bio) and solubilized by gentle rotation at $277 \mathrm{~K}$ for $1.5 \mathrm{~h}$. After the removal of unsolubilized materials by ultracentrifugation at $104000 \mathrm{~g}$ for $30 \mathrm{~min}$ at $277 \mathrm{~K}$, the supernatant was mixed with the P3NTA probe (produced inhouse; Backmark et al., 2013) and analyzed by fluorescencedetection size-exclusion chromatography (FSEC; Kawate \& Gouaux, 2006).

\subsection{Large-scale expression and purification of $\mathbf{P e p}_{\mathrm{So} 2}$}

The plasmid containing C-terminally $\mathrm{His}_{6}$-tag-fused $\mathrm{PepT}_{\text {So2 }}$ was introduced into E. coli Rosetta 2 (DE3) cells (Novagen). The transformed cells were grown in $181 \mathrm{LB}$ medium containing $50 \mu \mathrm{g} \mathrm{ml}^{-1}$ ampicillin at $310 \mathrm{~K}$. When the $A_{600}$ reached 0.5-0.8, expression was induced with $0.5 \mathrm{~m} M$ IPTG 
and the cells were grown for about $18 \mathrm{~h}$ at $293 \mathrm{~K}$. The cells were suspended in phosphate-buffered saline. The suspension was homogenized and mixed on ice, and was disrupted by 4-5 passes at $130 \mathrm{MPa}$ using a Microfluidizer (Microfluidics). After centrifugation at $25000 \mathrm{~g}$ for $20 \mathrm{~min}$ to remove the debris, the supernatant was ultracentrifuged at $125000 \mathrm{~g}$ for $1 \mathrm{~h}$ at $277 \mathrm{~K}$ using a Type 45 Ti rotor (Beckman Coulter). The membrane fraction was resuspended in membrane buffer (50 m $M$ Tris- $\mathrm{HCl}$ pH 8.0, $300 \mathrm{~m} M \mathrm{NaCl}, 0.5 \mathrm{~m} M$ TCEP, $10 \%$ glycerol) supplemented with cOmplete EDTA-free Protease Inhibitor Cocktail (Roche) and stored at $193 \mathrm{~K}$ until use.

The membrane fraction was solubilized in solubilization buffer supplemented with cOmplete EDTA-free Protease Inhibitor Cocktail (Roche) at $277 \mathrm{~K}$ for $2 \mathrm{~h}$. After removal of unsolubilized material by ultracentrifugation at $125000 \mathrm{~g}$ for $30 \mathrm{~min}$ at $277 \mathrm{~K}$ using a Type 45 Ti rotor (Beckman Coulter), the supernatant was mixed with $25 \mathrm{ml} \mathrm{Ni-NTA} \mathrm{Superflow}$ resin (Qiagen) equilibrated with $\mathrm{Ni}$ buffer $(50 \mathrm{~m} M$ Tris- $\mathrm{HCl}$ pH 8.0, $300 \mathrm{~m} M \mathrm{NaCl}, 0.5 \mathrm{~m} M$ TCEP, $5 \%$ glycerol, $0.05 \%$ DDM) containing $15 \mathrm{~m} M$ imidazole for $1 \mathrm{~h}$ at $277 \mathrm{~K}$. The mixture was transferred into an Econo-column (Bio-Rad) and the flowthrough fraction was collected. The resin was washed with ten column volumes of $\mathrm{Ni}$ buffer containing $50 \mathrm{mM}$ imidazole, and the protein sample was eluted with $\mathrm{Ni}$ buffer containing $300 \mathrm{~m} M$ imidazole. To cleave the $\mathrm{His}_{6}$ tag that was fused to the C-terminus of $\mathrm{PepT}_{\mathrm{So} 2}$, His-tagged TEV protease (produced in-house) was added to the eluted fraction to a 1:75(w:w) protease:protein ratio and the solution was dialyzed twice against 11 dialysis buffer $(50 \mathrm{mM}$ Tris- $\mathrm{HCl} \mathrm{pH} 8.0$, $300 \mathrm{~m} M \mathrm{NaCl}, 0.25 \mathrm{~m} M$ TCEP, $5 \%$ glycerol, $0.03 \%$ DDM). To remove the cleaved His tag and His-tagged TEV protease, the solution was mixed with Ni-NTA resin equilibrated with dialysis buffer for $1.5 \mathrm{~h}$ at $277 \mathrm{~K}$ and the collected flowthrough fraction was concentrated using an Amicon Ultra centrifugal filter (100 kDa molecular-weight cutoff; Millipore). The concentrated sample was ultracentrifuged at $40000 \mathrm{rev} \mathrm{\textrm {min } ^ { - 1 }}$ for $20 \mathrm{~min}$ using an S55A2 rotor (Hitachi Koki) and the supernatant was applied onto a HiLoad 16/600 Superdex 200 pg column (GE Healthcare) equilibrated with SEC buffer consisting of $50 \mathrm{~m} M$ Tris- $\mathrm{HCl} \mathrm{pH} 8.0,300 \mathrm{mM} \mathrm{NaCl}, 5 \%$ glycerol, $0.03 \%$ DDM. The purity and homogeneity of the protein sample were assessed by FSEC. The purity of the protein sample was also assessed by SDS-PAGE. The protein concentrations were estimated based on the absorbance at $280 \mathrm{~nm}\left(A_{280}\right)$ as measured using a NanoDrop spectrophotometer (Thermo Fisher Scientific). The protein bands detected in the SDS-PAGE gel were analyzed using the peptide mass fingerprint method.

\subsection{Expression and purification of saposin $A$}

The coding region of saposin A was cloned into a modified pET-28b vector that harbors an N-terminal $\mathrm{His}_{6}$ tag followed by the 'eTEV' sequence (Tabata et al., 2018), resulting in the protein sequence MGSSHHHHHHSSGLVPRENLYFQGM GSLPCDICKDVVTAAGDMLKDNATEEEILVYLEKTCD WLPKPNMSASCKEIVDSYLPVILDIIKGEMSRPGEVCS
ALNLCES. The protein cleaved by TEV protease represents the saposin A polypeptide sequence with three additional Nterminal residues (Gly-Met-Gly). The expression and purification of saposin A were performed according to a previously reported method (Frauenfeld et al., 2016).

\subsection{Reconstruction of $\mathrm{Pep}_{\mathrm{So} 2}$ into Salipro particles}

For the reconstruction of $\mathrm{PepT}_{\mathrm{So2} 2}$ into Salipro nanoparticles, $8 \mu \mathrm{l}$ of purified PepT $\mathrm{So}_{\mathrm{So} 2}$ at $20.4 \mathrm{mg} \mathrm{ml}^{-1}$ in TBSGD buffer (25 mM Tris- $\mathrm{HCl} \mathrm{pH}$ 8.0, 5\% glycerol, 0.03\% DDM), $30 \mu \mathrm{l}$ of a $5 \mathrm{mg} \mathrm{ml}^{-1}$ DMPG (Avanti) lipid solution in lipidsolubilization buffer (50 $\mathrm{m} M$ HEPES-NaOH pH 7.5, $150 \mathrm{~m} M$ $\mathrm{NaCl}, 0.224 \%$ DDM) and $12 \mu \mathrm{l}$ HBSD buffer $(50 \mathrm{mM}$ HEPES-NaOH pH 7.5, $150 \mathrm{mM} \mathrm{NaCl}, 0.03 \%$ DDM) were mixed and incubated at $298 \mathrm{~K}$ for $5 \mathrm{~min}$. Next, $120 \mu \mathrm{l}$ of purified saposin $\mathrm{A}$ at $1.2 \mathrm{mg} \mathrm{ml}^{-1}$ was added and the mixture was incubated at $278 \mathrm{~K}$ for $5 \mathrm{~min}$. Subsequently, $114 \mu \mathrm{l}$ saposin SEC buffer, consisting of $25 \mathrm{~m} M$ sodium phosphate $\mathrm{pH}$ 7.4, $200 \mathrm{mM} \mathrm{NaCl}$, was added to the mixture and incubated at $278 \mathrm{~K}$ for $5 \mathrm{~min}$, after which $234 \mu \mathrm{l}$ saposin SEC buffer was added. The mixture was injected into a Superdex 200 Increase 10/300 GL column (GE Healthcare) equilibrated with saposin SEC buffer. Fractions containing Salipro-PepT $\mathrm{S}_{\mathrm{So} 2}$ were pooled and concentrated to $\sim 0.5 \mathrm{mg} \mathrm{ml}^{-1}$ using an Amicon Ultra centrifugal filter ( $30 \mathrm{kDa}$ molecular-weight cutoff; Millipore).

\subsection{Cryo-EM sample preparation and data collection}

Quantifoil copper 200 mesh R1.2/1.3 holey carbon grids were glow-discharged on a glass slide for $30 \mathrm{~s}$. A $2.6 \mu \mathrm{l}$ aliquot of the sample solution was applied onto the grid and blotted by filter paper for $4.5 \mathrm{~s}$ at $100 \%$ humidity and $277 \mathrm{~K}$, and the grid was then quickly frozen by rapidly plunging it into liquid ethane using a Vitrobot Mark IV (Thermo Fisher Scientific). The grid was inserted into a Talos Arctica FEG transmission electron microscope (Thermo Fisher Scientific) operated at $200 \mathrm{kV}$, with the cryo-specimen stage cooled with liquid nitrogen. Cryo-EM images were recorded using a Falcon 3EC $4 \mathrm{k} \times 4 \mathrm{k}$ CMOS direct electron detector (Thermo Fisher Scientific) in counting mode at a nominal magnification of $\times 96000$, corresponding to an image pixel size of $0.87 \AA$, using the EPU software package. Movie frames of the $\mathrm{PepT}_{\mathrm{So} 2}$ tetramer embedded in Salipro were collected from $30^{\circ}$ to $50^{\circ}$ tilts of the stage in $10^{\circ}$ increments at a dose rate of $1.2 \mathrm{e} \mathrm{per}$ pixel per second and an exposure time of $68.38 \mathrm{~s}$. The total accumulated exposure of $82 \mathrm{e} \AA^{-2}$ was fractionated into 102 frames. A data set of 1609 micrographs ( $30^{\circ}$ tilt, 1275 micrographs; $40^{\circ}$ tilt, 272 micrographs; $50^{\circ}$ tilt, 62 micrographs) was collected in a single session using a defocus range between 1.0 and $3.0 \mu \mathrm{m}$.

\subsection{Image processing}

Data sets from the various tilts from $30^{\circ}$ to $50^{\circ}$ were used. The movie frames were subsequently aligned to correct for beam-induced movement and drift using MotionCor2 (Zheng et al., 2017), and the parameters for the contrast transfer 
Table 2

Crystallization conditions.

\begin{tabular}{|c|c|c|}
\hline & Form $A$ crystal & Form $B$ crystal \\
\hline Method & Lipidic cubic phase (LCP) & Lipidic cubic phase (LCP) \\
\hline Plate type & 96-well glass sandwich plate & 96-well glass sandwich plate \\
\hline Temperature (K) & 293 & 293 \\
\hline Buffer composition of protein solution & $\begin{array}{l}300 \mathrm{~m} M \mathrm{NaCl}, 50 \mathrm{~m} M \text { Tris- } \mathrm{HCl} \mathrm{pH} \text { 8.0, } 5 \% \text { glycerol, } \\
0.03 \% \text { DDM }\end{array}$ & $\begin{array}{l}300 \mathrm{~m} M \mathrm{NaCl}, 50 \mathrm{~m} M \text { Tris- } \mathrm{HCl} \mathrm{pH} 8.0,5 \% \text { glycerol, } \\
0.03 \% \mathrm{DDM}\end{array}$ \\
\hline Composition of reservoir solution & $\begin{array}{l}40 \% \text { PEG } 200,100 \mathrm{~m} M \text { sodium malonate } \mathrm{pH} 7.0, \\
100 \mathrm{~m} M \text { sodium acetate } \mathrm{pH} 4.0\end{array}$ & $40 \%$ PEG $200,100 \mathrm{~m} M \mathrm{NaCl}, 100 \mathrm{~m} M$ sodium acetate $\mathrm{pH} 4.0$ \\
\hline
\end{tabular}

Table 3

$\mathrm{X}$-ray data-collection and refinement statistics.

Values in parentheses are for the outer shell.

\begin{tabular}{|c|c|c|}
\hline & $\begin{array}{l}\text { Form } A \text { crystal } \\
\text { (PDB entry } 6 \mathrm{jkd})\end{array}$ & $\begin{array}{l}\text { Form } B \text { crystal } \\
\text { (PDB entry } 6 \mathrm{jkc})\end{array}$ \\
\hline \multicolumn{3}{|l|}{$\mathrm{X}$-ray data collection } \\
\hline Diffraction source & BL32XU, SPring-8 & BL32XU, SPring-8 \\
\hline Wavelength $(\AA)$ & 1.0000 & 1.0000 \\
\hline Temperature $(\mathrm{K})$ & 100 & 100 \\
\hline Detector & $\begin{array}{l}\text { EIGER X 9M, } \\
\text { Dectris }\end{array}$ & $\begin{array}{l}\text { EIGER X 9M, } \\
\text { Dectris }\end{array}$ \\
\hline $\begin{array}{l}\text { Crystal-to-detector distance } \\
\quad(\mathrm{mm})\end{array}$ & 300 & 280 \\
\hline Rotation range per image $\left(^{\circ}\right)$ & 0.1 & 0.1 \\
\hline Rotation range per crystal $\left({ }^{\circ}\right)$ & 10 & 5 \\
\hline No. of crystals & 10 & 9 \\
\hline Space group & I4 & $P 42_{1} 2$ \\
\hline$a, b, c(\AA)$ & $115.1,115.1,110.1$ & $119.4,119.4,104.3$ \\
\hline Resolution range $(\AA)$ & $50-3.90(4.14-3.90)$ & $47.53-3.50(3.71-3.50)$ \\
\hline Total No. of reflections & 23358 & 29586 \\
\hline No. of unique reflections & 6448 & 8312 \\
\hline Completeness (\%) & $97.4(97.8)$ & $83.0(83.4)$ \\
\hline Multiplicity & $3.6(3.7)$ & $3.6(3.5)$ \\
\hline$\langle I / \sigma(I)\rangle$ & $4.38(1.44)$ & $5.49(1.22)$ \\
\hline$R_{\text {meas }}$ & $0.455(1.277)$ & $0.328(1.586)$ \\
\hline $\mathrm{CC}_{1 / 2}$ & $0.946(0.459)$ & $0.961(0.293)$ \\
\hline \multicolumn{3}{|l|}{ Refinement } \\
\hline Resolution $(\AA)$ & $46.65-3.90$ & $47.53-3.50$ \\
\hline$R_{\text {work }} / R_{\text {free }}$ & $0.2524 / 0.2832$ & $0.2672 / 0.3030$ \\
\hline \multicolumn{3}{|l|}{ No. of atoms } \\
\hline Protein & 3476 & 3498 \\
\hline Ligand & 0 & 0 \\
\hline Solvent & 0 & 0 \\
\hline \multicolumn{3}{|l|}{ Average $B$ factors $\left(\AA^{2}\right)$} \\
\hline Protein & 70.3 & 65.7 \\
\hline Ligand & - & - \\
\hline Solvent & - & - \\
\hline \multicolumn{3}{|l|}{ R.m.s. deviations } \\
\hline Bond lengths $(\AA)$ & 0.002 & 0.003 \\
\hline Bond angles $\left({ }^{\circ}\right)$ & 0.51 & 0.55 \\
\hline \multicolumn{3}{|l|}{ Ramachandran plot } \\
\hline Favored $(\%)$ & 97.1 & 96.9 \\
\hline Allowed (\%) & 2.9 & 3.1 \\
\hline Outliers (\%) & 0 & 0 \\
\hline
\end{tabular}

function (CTF) were estimated using Gctf (Zhang, 2016). A total of 288417 particle images were automatically picked from 1484 micrographs using Gautomatch (http:// www.mrc-lmb.cam.ac.uk/kzhang/), and two-dimensional (2D) and three-dimensional (3D) classifications were then performed using Relion-3.0 (Zivanov et al., 2018). Particle images from the good 2D class were selected to obtain the initial 3D model of the $\mathrm{PepT}_{\mathrm{So} 2}$ tetramer embedded in Salipro using cryoSPARC (Punjani et al., 2017) with C4 symmetry. A total of 43172 particles from the best 3D class were subjected to $3 \mathrm{D}$ refinement, which produced a reconstruction with a resolution of $5.9 \AA$ and a $B$ factor of $-307 \AA^{2}$. The 3D-refined structure was further refined with per-particle defocus with a search range of $\pm 2000 \AA$ and Bayesian polishing, which improved the resolution to $4.3 \AA$ with a $B$ factor of $-171 \AA^{2}$. To improve the resolution further, CTF refinement with beamtilt correction and Bayesian polishing was iterated two times, giving $4.3 \AA$ resolution and a $B$ factor of $-161 \AA^{2}$ and finally $4.1 \AA$ resolution and a $B$ factor of $-147 \AA^{2}$. The 3D density map was visualized using UCSF Chimera (Pettersen et al., 2004).

\subsection{X-ray crystallographic analyses}

The PepT $\mathrm{T}_{\mathrm{So} 2}$ sample was mixed with monoolein $(\mathrm{Nu}-\mathrm{Ch}$-k Prep) at a 2:3(w:w) protein:lipid ratio using coupled syringes. Drops of the mixture $(30 \mathrm{nl})$ were dispensed onto 96-well Laminex glass sandwich plates (MD11-50-100; Molecular Dimensions) and overlaid with $800 \mathrm{nl}$ reservoir solution using a Gryphon LCP crystallization robot (Art Robbins). The initial crystallization screening was performed at $293 \mathrm{~K}$ using in-house-produced grid-screening crystallization kits as reservoir solutions. To optimize the crystallization conditions, StockOptions Salt and Additive Screen (Hampton Research) was added to the reservoir solutions for LCP crystallization, in addition to optimization of the $\mathrm{pH}$ and the concentrations of precipitants and salts. Crystals were picked up using MicroMeshes (MiTeGen), MicroMounts (MiTeGen) or LithoLoops (Protein Wave) and were flash-cooled in liquid nitrogen. Crystallization information is provided in Table 2.

\subsection{X-ray data collection and processing}

All X-ray diffraction data sets were collected by the helical data-collection method using the microfocus beam at SPring- 8 beamline BL32XU (Hirata et al., 2013). The locations of well diffracting crystals were identified by raster scanning, and a $5^{\circ}$ or $10^{\circ}$ wedge of data was collected from each crystal. All diffraction data were processed with $X D S$ (Kabsch, 2010) and were merged with $X S C A L E$ based on hierarchical clustering analysis with the cross-correlation method implemented in the KAMO software (Yamashita et al., 2018). 
Table 4

Cryo-EM data-collection and refinement statistics.

\begin{tabular}{|c|c|c|c|c|}
\hline & \multicolumn{4}{|c|}{$\mathrm{PepT}_{\mathrm{So2} 2}$ embedded in Salipro nanoparticles (PDB entry 6ji1, EMDB ID EMD-9832) } \\
\hline & $0^{\circ}$ tilt & $30^{\circ}$ tilt & $40^{\circ}$ tilt & $50^{\circ}$ tilt \\
\hline \multicolumn{5}{|l|}{ Cryo-EM data collection } \\
\hline Accelerating voltage $(\mathrm{kV})$ & 300 & 200 & & \\
\hline Camera & Falcon III (counting) & Falcon I & & \\
\hline Total dose $\left(\mathrm{e} \AA^{-2}\right)$ & 60 & 82 & & \\
\hline No. of micrographs & 864 & 1275 & 272 & 62 \\
\hline Resolution $(\AA)$ & 6.7 & 4.13 & & \\
\hline Map-sharpening $B$ factor $\left(\AA^{2}\right)$ & -200 & -147 & & \\
\hline Fourier shell correlation criterion & 0.143 & 0.143 & & \\
\hline Particles used in final 3D refinement & 3415 & 43172 & & \\
\hline \multicolumn{5}{|l|}{ Coordinate refinement and validation } \\
\hline \multicolumn{5}{|l|}{ R.m.s. deviations } \\
\hline Bond lengths $(\AA)$ & & 0.010 & & \\
\hline MolProbity score & & 2.09 & & \\
\hline Clashscore (all-atom) & & 7.36 & & \\
\hline
\end{tabular}

Molecular replacement was performed with Phaser (McCoy et al., 2007). For structure determination of the form $B$ crystal, the structure of molecule $A$ of $\mathrm{PepT}_{\mathrm{So} 2}$ (PDB entry 4lep), reported as a dimeric form, was used as the search model. For structure determination of the form $A$ crystal, the structure determined from the form $B$ crystal (PDB entry 6jkc) was used as the search model. Model building was performed with Coot (Emsley \& Cowtan, 2004; Emsley et al., 2010). Refinement was performed with phenix.refine (Adams et al., 2010) and REFMAC5 (Murshudov et al., 2011) using the CCP4i2 interface (Potterton et al., 2018). The data-collection statistics are summarized in Table 3. Molecular graphics were generated using CueMol (http://www.cuemol.org).

\subsection{Model building for cryo-EM}

The crystal structure of $\mathrm{PepT}_{\mathrm{So} 2}$ determined in this work (form $B$ crystal) was fitted as a rigid body into the 3D density map using UCSF Chimera and Coot (Emsley \& Cowtan, 2004; Emsley et al., 2010). Refinement was performed with phenix.refine (Adams et al., 2010). The conformations were refined using real-space refinement in PHENIX (Adams et al., 2010) with secondary-structure restraints. We first performed model refinement for each subunit separately against the corresponding EM maps. To resolve the possible clashes in the subunit interfaces, we refined the entire models against the corresponding EM maps. Table 4 summarizes the refinement statistics for the overall structure, the deposited maps and their associated coordinates.

\section{Results}

\subsection{FSEC-based optimization of expression conditions}

For structural analysis of tetrameric $\mathrm{PepT}_{\mathrm{So} 2}$, the expression and purification conditions were optimized. Full-length $\mathrm{Pep}_{\mathrm{So} 2}$ fused with a His tag at the C-terminus was expressed using a modified pET vector (Nishizawa et al., 2013; Table 1), and the expression level and homogeneity of the samples were evaluated by an FSEC-based high-throughput screening method (Kawate \& Gouaux, 2006) using the P3NTA peptide as a probe (Backmark et al., 2013). Unexpectedly, the number of histidine residues fused to the C-terminus drastically affected the oligomerization state of $\mathrm{PepT}_{\mathrm{So} 2}$, and the $\mathrm{His}_{6}$-tagged $\mathrm{PepT}_{\text {So2 }}$ formed a tetramer more readily (Fig. $1 a$ ). Under the conditions that we tested, the expression levels of PepT $\mathrm{T}_{\mathrm{So} 2}$ were greater in the BL21 (DE3) derivative strain of E. coli compared with the C41 (DE3) strain, which was used in the previous studies (Guettou et al., 2013). The presence or absence of tRNAs reading rare codons in the expression host did not affect the expression level. The addition of glucose to the medium did not affect the expression level. The expression level of PepT $\mathrm{T}_{\mathrm{So} 2}$ was higher when using LB medium compared with the TB medium used in the previous study. These culture conditions affected the expression level but not the oligomerization state of $\mathrm{PepT}_{\mathrm{So} 2}$.

\subsection{Large-scale expression and purification of $\mathrm{Pep}_{\mathrm{So} 2}$}

C-terminally $\mathrm{His}_{6}$-tagged $\mathrm{PepT}_{\mathrm{So} 2}$ was expressed on a large scale under the conditions optimized in this study. PepT $\mathrm{T}_{\mathrm{So} 2}$ solubilized with DDM was purified by Ni-NTA affinity chromatography followed by cleavage of the His tag, a second Ni-NTA chromatography step to remove the His-tagged TEV protease and size-exclusion chromatography (SEC). The SEC chromatogram peak showed monodispersity, and the peak fractions showed a single band in the SDS-PAGE analysis, demonstrating high purity and homogeneity of the obtained PepT $_{\text {So2 }}$ sample (Figs. $1 b$ and $1 c$ ). The elution volume of $\mathrm{PepT}_{\mathrm{So} 2}$ in the SEC chromatogram was $14.8 \mathrm{ml}$ in DDMcontaining buffer using a Superose 6 Increase column (GE 
Healthcare) (Fig. 1b), which was a reasonable elution position for tetrameric $\mathrm{PepT}_{\mathrm{So} 2}$ (the molecular weight is about $200 \mathrm{kDa}$ ). The final yield was about $2.5 \mathrm{mg}$ per litre of $E$. coli culture.

\subsection{Single-particle cryo-EM analyses of PepT $\mathrm{T}_{\mathrm{so} 2}$}

To gain structural insight into oligomer formation by $\mathrm{PepT}_{\mathrm{So} 2}$, we reconstructed PepT $\mathrm{T}_{\mathrm{So} 2}$ into Salipro nanoparticles using saposin A and DMPG lipids, and performed singleparticle analysis using cryo-electron microscopy (Figs. $2 a-2 e$, Supplementary Fig. S1). Although square-shaped particles were clearly observed in the cryo-EM micrographs of Pep $\mathrm{T}_{\mathrm{So} 2}$ reconstructed in Salipro, the overall resolution was limited to $6.7 \AA$ and the quality of the map was too poor to refine the atomic model owing to severe orientation bias (Supplementary Fig. S1). Interestingly, this preferred specimen-orientation problem was not reported in the previous study (Frauenfeld et al., 2016). In the previous study, bovine brain lipid extract was used for reconstruction, but this product is no longer commercially accessible. The difference in the lipids used for reconstruction in this study might affect the physical properties of the specimen under solution conditions and thus cause the preferred specimen-orientation problem. To alleviate the orientation bias, we tried to collect data by tilting the grid from $30^{\circ}$ to $50^{\circ}$ (Tan et al., 2017), which drastically improved the data quality (Supplementary Fig. S1). A total of 43172 selected particles from three different tilt angles $\left(30^{\circ}, 40^{\circ}\right.$ and $50^{\circ}$ ) yielded a 3D EM map at an overall resolution of $4.1 \AA$, according to the gold-standard Fourier shell correlation (FSC) 0.143 criterion (Scheres \& Chen, 2012; Fig. $2 d$, Supplementary Fig. S2, Table 4). The tetrameric organization of $\mathrm{PepT}_{\mathrm{So} 2}$ is clearly visualized in the final 3D density map (Fig. $2 f$ ), in which the secondary-structural features and the side-chain densities from bulky amino-acid residues are clearer compared with the previous study ( $6.5 \AA$ resolution; Frauenfeld et al.,
2016; Fig. 2g). Residual density derived from the Salipro scaffold composed of saposin A and lipids was also observed around the tetrameric $\mathrm{PepT}_{\mathrm{So} 2}$ molecule; however, the density in these regions was not so clear compared with the PepT $\mathrm{T}_{\mathrm{So} 2}$ molecule (Fig. $2 h$ ), suggesting flexibility of the Salipro scaffold region. The EM density map suggested a contribution of the extracellular loops between the TM helices that were not well resolved in the previous study to the tetramer assembly (Figs. $2 h$ and $2 f$ ). However, the atomic-level oligomerization mechanism remained unclear because of the resolution limit.

\subsection{X-ray crystallographic analyses of Pep $\mathrm{T}_{\mathrm{So} 2}$}

To further improve the resolution, we tried to crystallize tetrameric PepT $\mathrm{S}_{\mathrm{So} 2}$ using the LCP method (Caffrey \& Cherezov, 2009) at protein concentrations of 20,45 and $75 \mathrm{mg} \mathrm{ml}^{-1}$. Microcrystals of PepT $T_{\text {So2 }}$ were obtained using a $45 \mathrm{mg} \mathrm{ml}^{-1}$ sample under multiple conditions containing PEG 200 as a precipitant (Fig. 3). Since the size of the crystals was too small and data collection from a single crystal was impossible, small wedge data were collected from microcrystals using the microfocus beam at BL32XU at SPring-8, Hyogo, Japan and were combined using the multi-crystal merging software KAMO (Yamashita et al., 2018). By using the molecularreplacement method with the previously reported structure of the $\mathrm{PepT}_{\mathrm{So} 2}$ dimer (PDB entry 4lep, molecule $A$ ) as the search model (Guettou et al., 2014), the structures of the form $A$ and $B$ crystals were finally determined at resolutions of 3.9 and $3.5 \AA$, respectively (Table 3 ).

\subsection{Overall structure of tetrameric Pep $\mathrm{T}_{\mathrm{So} 2}$}

The PepT $\mathrm{T}_{\mathrm{So} 2}$ structures obtained from the two crystal forms (forms $A$ and $B$ ) were assembled as tetramers in similar manners (r.m.s.d. of $0.83 \AA$ for all $\mathrm{C}^{\alpha}$ atoms), although the space groups and the crystal packings were different (Table 3 , Supplementary Fig. S3). Thus, in the following we focus on the

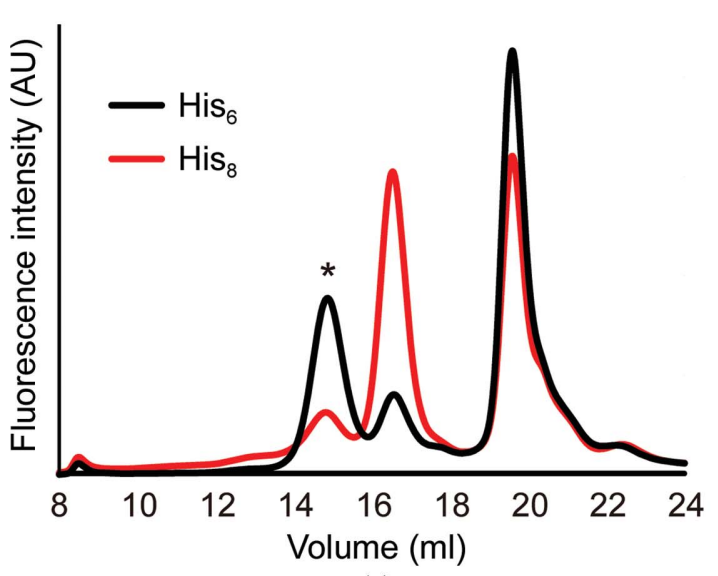

(a)

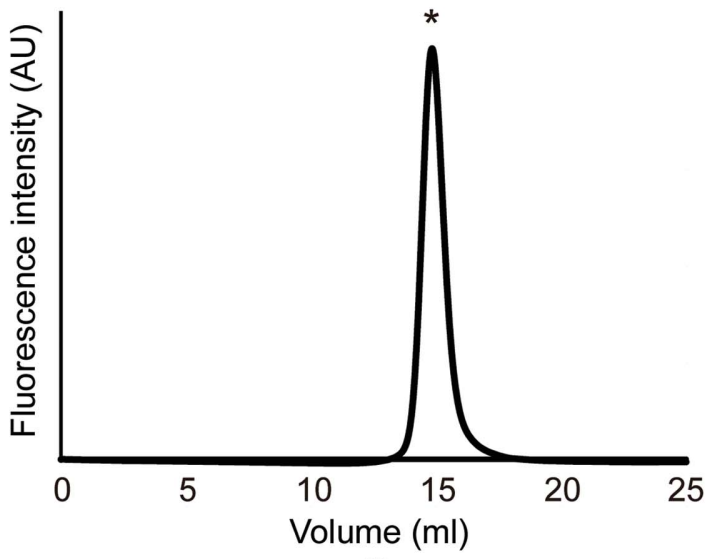

(b)

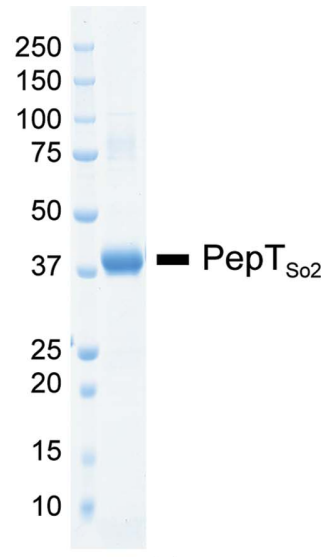

(c)

Figure 1

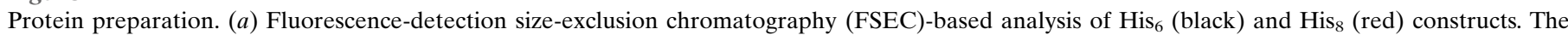
histidine tag-specific fluorescent probe P3NTA was used for detection (excitation, $482 \mathrm{~nm}$; emission, $520 \mathrm{~nm}$ ). The peak corresponding to tetrameric

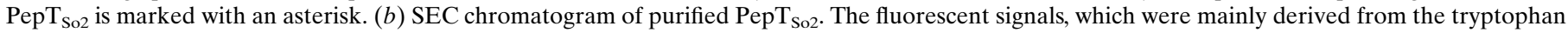
residues, were monitored by the fluorescence detector, with excitation at $280 \mathrm{~nm}$ and emission at $350 \mathrm{~nm}$. The peak corresponding to tetrameric PepT So2 $_{2}$ is marked with an asterisk. (c) SDS-PAGE analysis with Coomassie Brilliant Blue staining. Left lane, molecular-weight markers (labeled in kDa); right lane, the merged peak fraction from the SEC purification. 
structure obtained from the form $B$ crystal (Figs. $4 a, 4 b$ and $4 c$ ), as the quality of its electron density is better than that of the other crystal form. All of the protomers in the tetramer adopted the inward-open conformation, in which a large central hydrophilic substrate-binding pocket faces the intracellular side of the membrane. The structural comparison shows that the present tetrameric structure superimposes well with the previously reported dimeric structure at the protomer

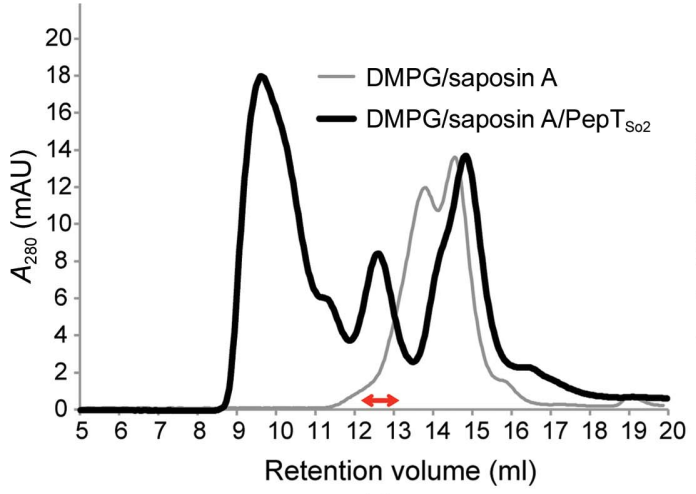

(a)

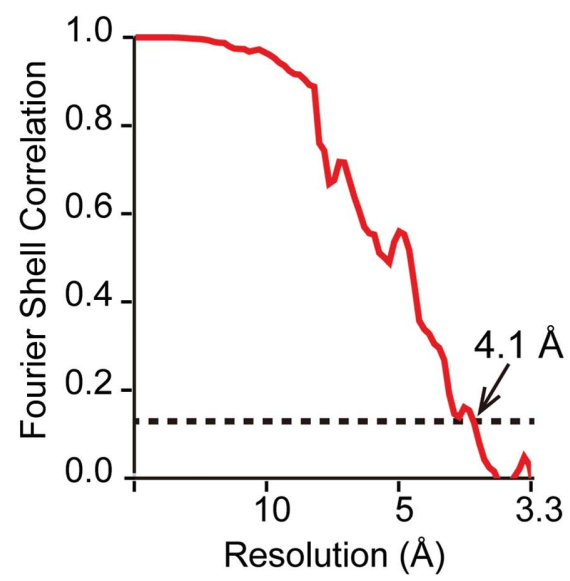

(d)

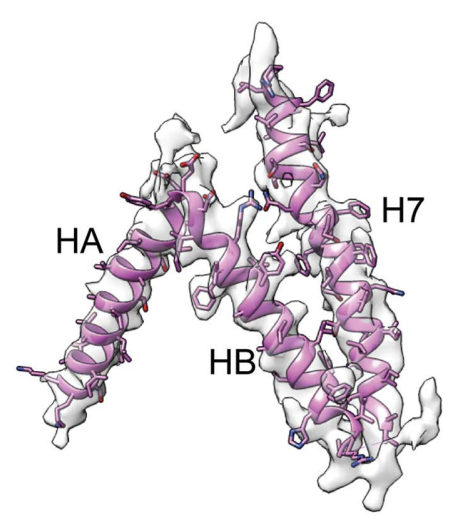

$(g)$

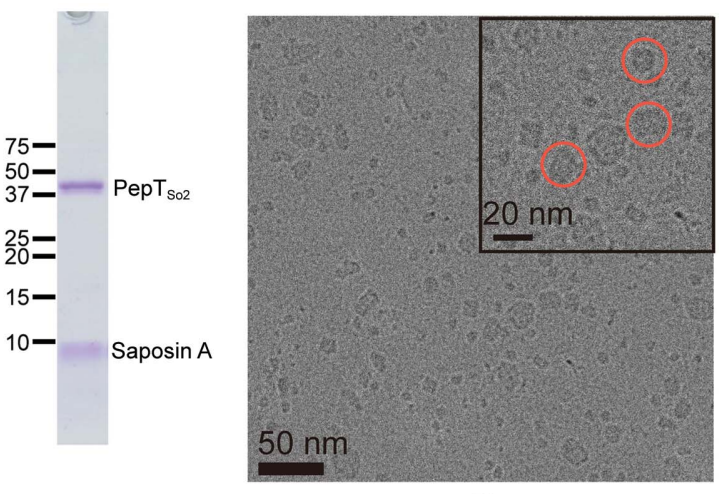

(b)

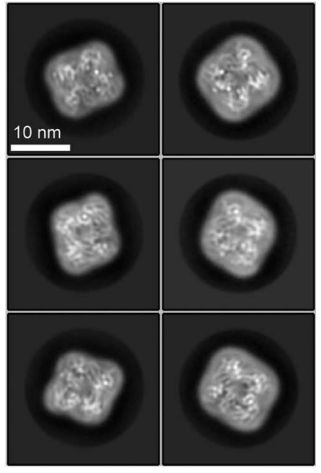

(c)

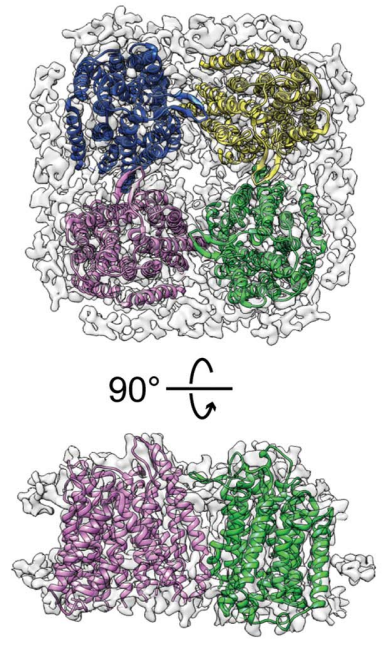

(f)

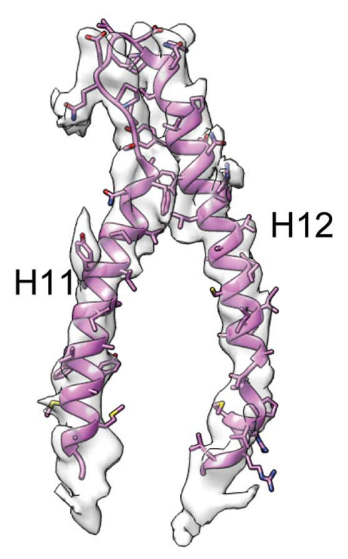

)

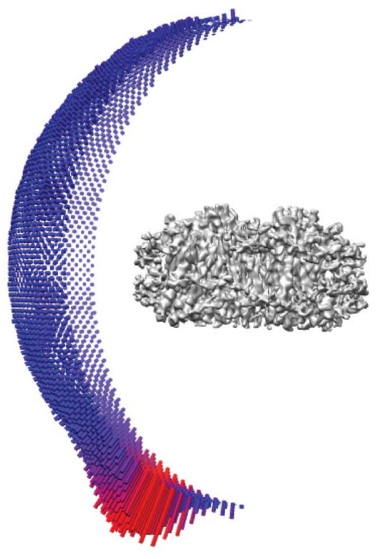

(e)



(h)

Figure 2

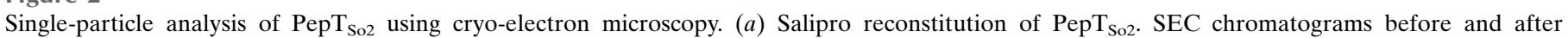
reconstitution of $\mathrm{PepT}_{\mathrm{So} 2}$ are shown as gray and black lines, respectively (left panel). The peak fractions indicated by the red arrow were analyzed by SDS-PAGE (right panel). (b) Raw electron micrograph of PepT $\mathrm{So2}_{\mathrm{S}}$. The Salipro-reconstituted PepT $\mathrm{S}_{\mathrm{So2}}$ particles are indicated by red circles. $(c)$ Representative $2 \mathrm{D}$ class averages generated from the particles. The scale bar represents $10 \mathrm{~nm}$. (d) Gold-standard FSC between two independently refined half-maps. (e) Euler angle distribution of all particles collected by the tilting method. $(f) 3 \mathrm{D}$ density maps of the tetrameric PepT $\mathrm{S}_{\mathrm{So} 2}$ incorporated in the Salipro nanoparticle. The atomic model was refined by using the crystal structure determined in this study as the initial model. $(g)$ Densities of selected helices and side chains. $(h)$ The density around the inter-protomer interaction area. The extracellular loop of a protomer is colored green and $\mathrm{H} 12$ of the neighboring protomer is colored magenta. 


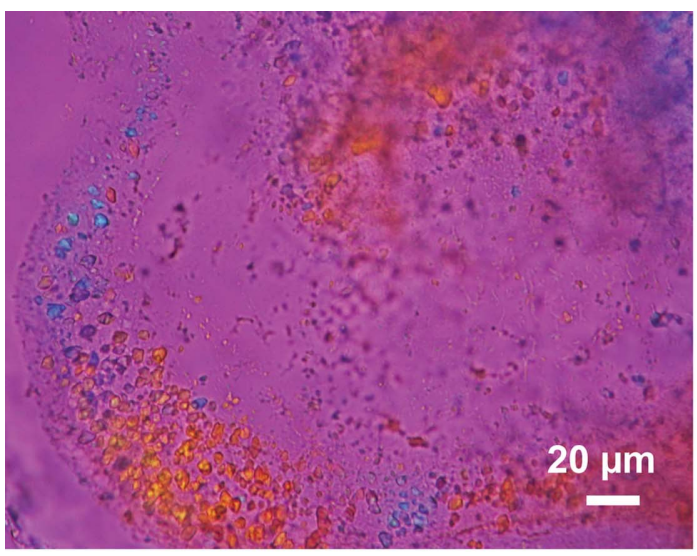

(a)

Figure 3

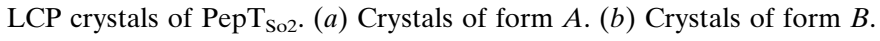

level (r.m.s.d. of $1.1 \AA$ for all $\mathrm{C}^{\alpha}$ atoms; Supplementary Fig. $\mathrm{S} 4 a$ ). The present crystal structures fitted well to the cryo-EM map obtained in this study (Fig. 2f, Supplementary Fig. 4b).

\subsection{Intermolecular interactions of the $\mathrm{Pep}_{\mathrm{So} 2}$ tetramer}

In the structure determined in this study, a sliding motion of H12 was also observed compared with the previously reported dimeric structure (Supplementary Fig. 4a), supporting the importance of $\mathrm{H} 12$ for tetramer formation suggested in the previous report (Guettou et al., 2014). On the intracellular side, inter-subunit hydrophobic interactions were formed between $\mathrm{H} 12$ of one protomer and $\mathrm{H} 5$ and $\mathrm{H} 8$ of the neighboring protomer (Fig. 4c). On the extracellular side of the present structure, electron density for the extracellular loop (ECL), which was not well resolved in the previous structures, was clearly observed (Fig. 4f). The ECL consists of two extracellular linkers between $\mathrm{H} 7-\mathrm{H} 8\left(\mathrm{ECL}_{\mathrm{a}}\right)$ and $\mathrm{H} 9-\mathrm{H} 10$ $\left(\mathrm{ECL}_{\mathrm{b}}\right.$ ) (Figs. $4 d$ and $4 e$ ), in which four $\beta$-strands form parallel and antiparallel $\beta$-sheet structures. The structure suggested that the ECL plays an important role in $\mathrm{PepT}_{\mathrm{So} 2}$ tetramer formation, together with $\mathrm{H} 12$ (Figs. $4 d$ and $4 e$ ). The ECL interacts with $\mathrm{H} 9$ and $\mathrm{H} 12$ of the neighboring protomer by hydrophobic or hydrogen-bond interactions (Figs. $4 a, 4 d$ and $4 e$ ). In the previous study, the importance of Asn468 in H12 for the tetramerization of PepT $\mathrm{T}_{\mathrm{So} 2}$ was suggested (Guettou et al., 2014). The higher resolution structure determined in this study clearly shows that the side chains of Asn465 and Asn468 in $\mathrm{H} 12$ form hydrogen bonds to the ECL of the neighboring protomer, thus stabilizing the tetramer (Figs. $4 a, 4 d$ and $4 e$ ). These two asparagine residues and the amino-acid sequence of the ECL are not conserved among the POT family members. Interestingly, the ECL of $\mathrm{PepT}_{\mathrm{So} 2}$ is longer compared with those of other prokaryotic orthologues with structures that were reported to be monomers or dimers (Fig. $4 g$ ). In contrast, among the POT family members that are phylogenetically closer to $\mathrm{PepT}_{\mathrm{So} 2}$ at the amino-acid level, the peptide lengths of the ECL are preserved, while the amino-acid sequences are not highly conserved. These results suggest that these nonconserved amino-acid residues play an important role in

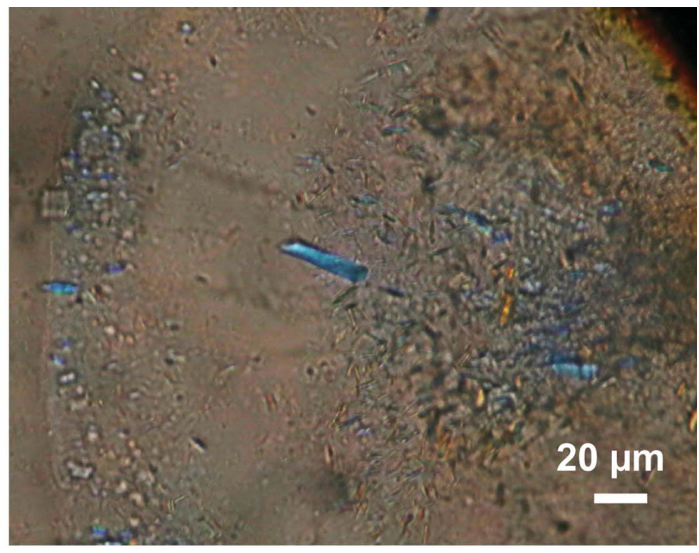

(b)

$\mathrm{PepT}_{\mathrm{So} 2}$-specific tetramer formation, which is further supported by the mutational analyses using FSEC (Fig. 4h).

\section{Discussion}

Many membrane proteins exist and function as oligomers in the membrane. The oligomeric states are highly diverse among the individual membrane proteins, and are sometimes in equilibrium depending on the protein concentration in the membrane. For membrane transporters, it has been suggested that oligomerization plays various roles, such as in transport activity, membrane trafficking, regulation of function and turnover (Alguel et al., 2016). Oligomerization can allow transporters to form a stable functional structure. In some cases, such as ATP-binding cassette (ABC) transporters (Locher, 2016) and the small multidrug transporter EmrE (Chen et al., 2007), the interface of the protomers forms the substrate-binding site and translocation pathway. Another example is the well studied bacterial glutamine transporter $\mathrm{Glt}_{\mathrm{ph}}$, which assembles as a trimer regardless of concentration, although each protomer contains the substrate-transport pathway (Yernool et al., 2004). Previous functional and structural analyses have suggested that the trimeric arrangement of $\mathrm{Glt}_{\mathrm{ph}}$ plays a critical role in conformational changes during the transport cycle (Reyes et al., 2009). In the above cases, the manner of oligomeric arrangement is widely preserved at the family level and is critical for proper function.

In contrast, there are some membrane transporters that form species-specific or subtype-specific oligomers, in which each protomer consists of a transport unit. This study provides structural insight into one of these types of transporters, $\mathrm{PepT}_{\text {So2 }}$, which belongs to the POT family of the MFS. $\mathrm{PepT}_{\text {So2 }}$ is derived from the Gram-negative anaerobic bacterium $S$. oneidensis and is known to assemble as a tetramer (Guettou et al., 2014). In contrast, another POT family protein from $S$. oneidensis, $\mathrm{Pep}_{\mathrm{So}}$, exists as a monomer in solution (Guettou et al., 2014). Previous reports also revealed that other POT family members exist as mixtures of monomers and dimers, while tetramer formation has only 

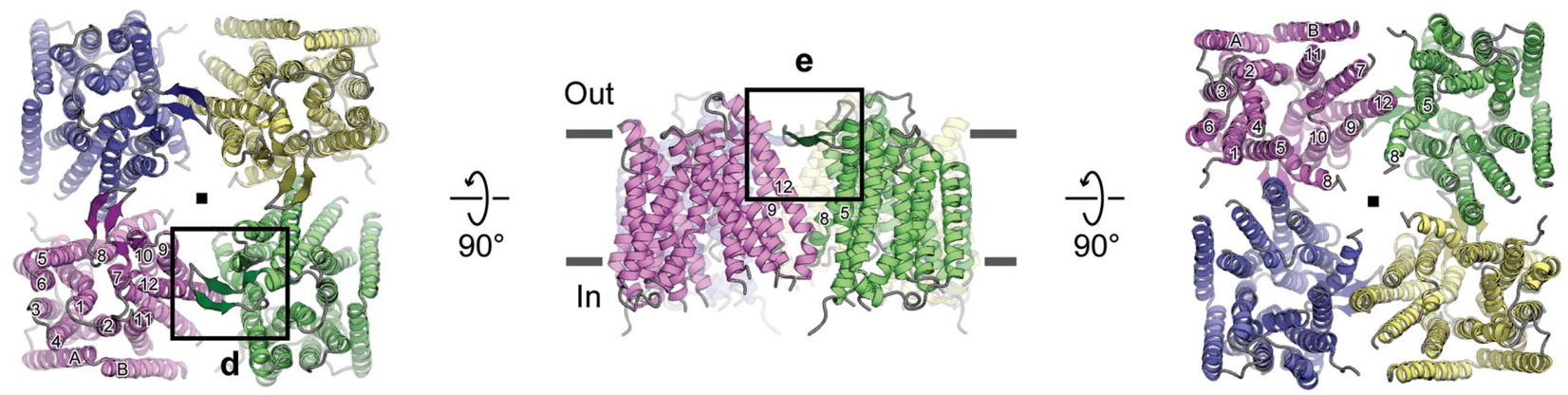

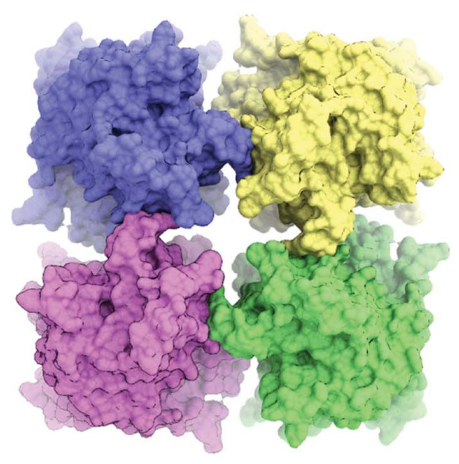

(a)

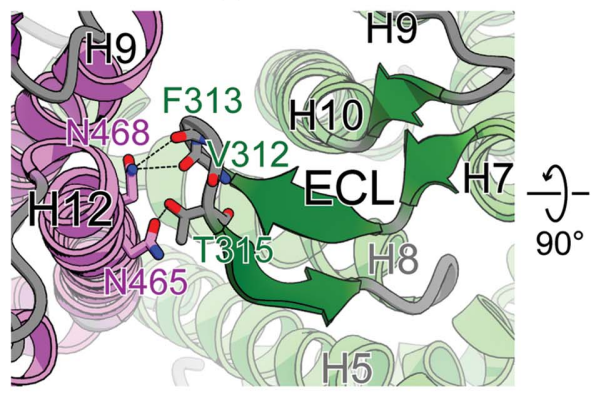

(d)

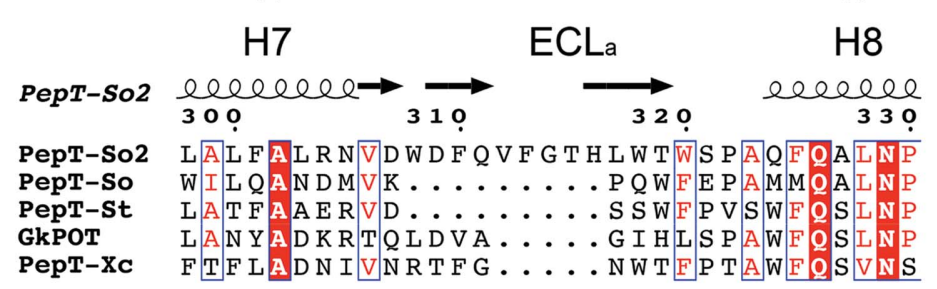

\begin{tabular}{|c|c|c|}
\hline & ECLb & H10 \\
\hline РepT-So2 & $\begin{array}{r}\text { elebelee } \\
380\end{array}$ & $\begin{array}{l}\text { elebelecele } \\
390 \\
\mathbf{3}\end{array}$ \\
\hline $\begin{array}{l}\text { PepT-So2 } \\
\text { PepT-So } \\
\text { PepT-St } \\
\text { GkPOT } \\
\text { PepT-XC }\end{array}$ & 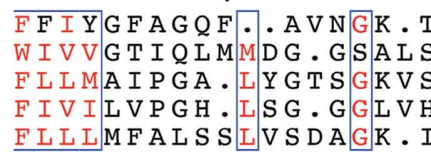 & 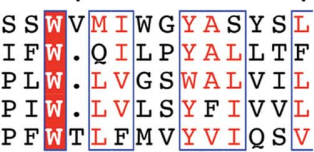 \\
\hline
\end{tabular}

(g)

(b)

(e)

$\mathrm{H} 8$

N
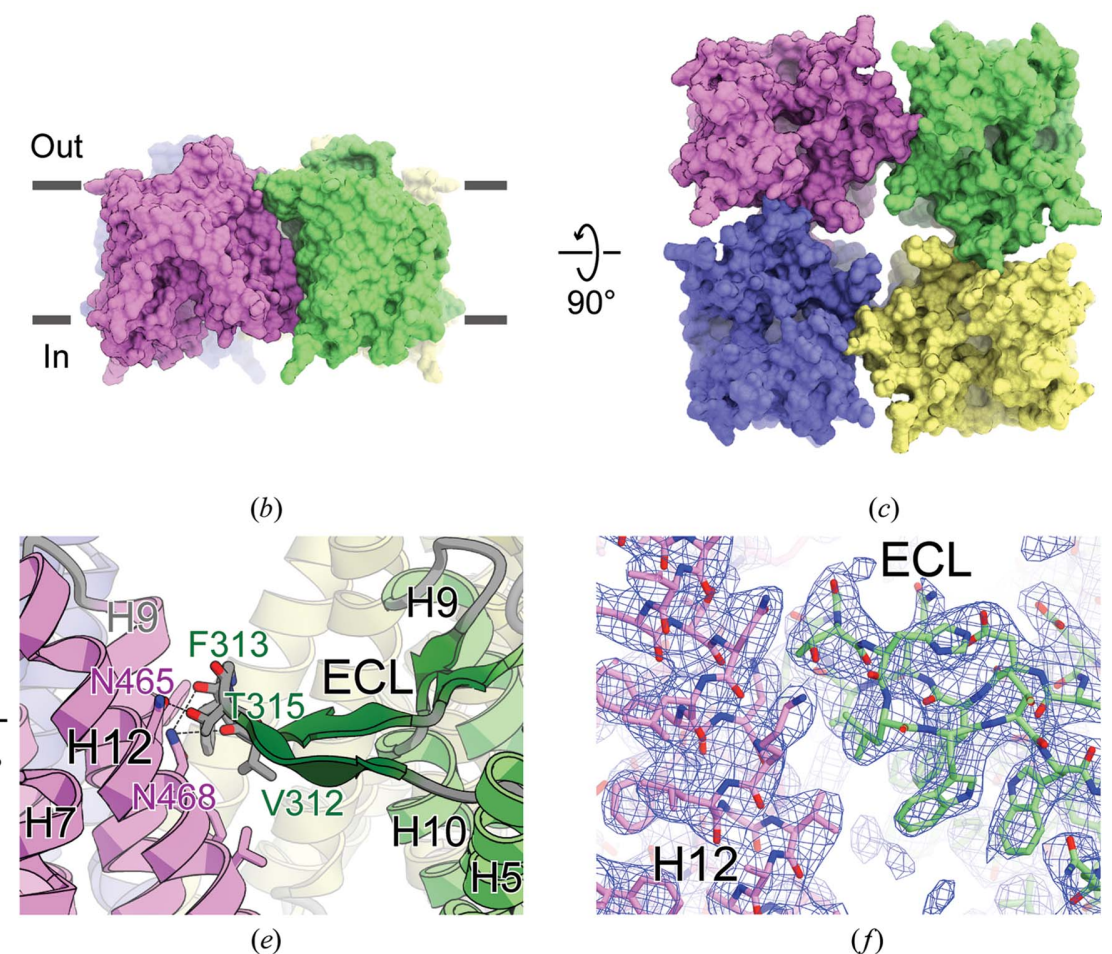

(c)

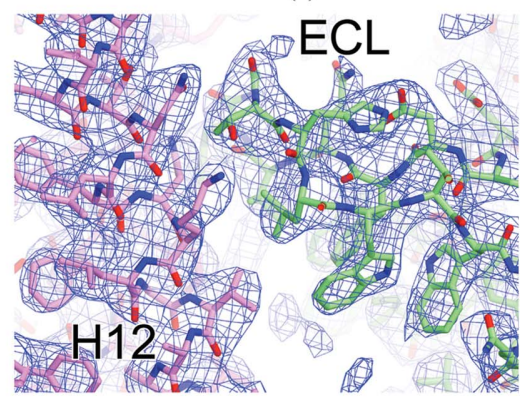

(f)

WT

N465A

N468A

$\triangle \mathrm{ECL}-1(\Delta 312-317)$

$\triangle E C L-2(\Delta 308-313)$

$\triangle$ ECL-3 ( $\triangle 310-318)$

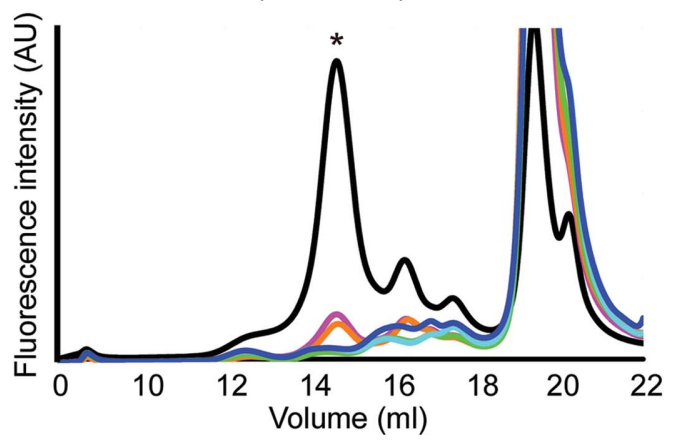

(h)

Figure 4

Tetrameric organization of $\operatorname{PepT}_{\mathrm{So} 2} \cdot(a, b, c)$ The overall structure of tetrameric $\mathrm{PepT}_{\mathrm{So} 2}$ is viewed from the $(a)$ extracellular, $(b)$ membrane and $(c)$ intracellular sides. The four protomers are colored magenta, green, blue and yellow. The rectangles labeled 'd' and 'e' indicate the regions highlighted in $(d)$ and $(e)$, respectively. $(d, e)$ The intermolecular interactions of the extracellular loop (ECL) with H12 of the neighboring protomer are viewed from the $(d)$ extracellular and $(e)$ membrane sides. The possible hydrogen bonds of up to $3.2 \AA$ are shown as black dotted lines. $(f)$ A $2 m F_{\mathrm{o}}-D F_{\mathrm{c}}$ electrondensity map of the interacting area between the ECL and H12 contoured at $1.1 \sigma$. The $\mathrm{C}$ atoms of the two protomers are colored magenta and green. ( $g$ )

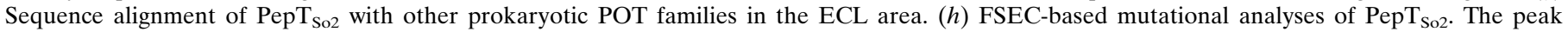
corresponding to tetrameric $\mathrm{PepT}_{\mathrm{So} 2}$ is marked with an asterisk. 
been observed for PepT $\mathrm{T}_{\mathrm{So2}}$ (Guettou et al., 2014; Löw et al., 2013). In this study, a combination of X-ray crystallographic and single-particle cryo-EM analyses revealed the speciesspecific tetramer formation mechanism of $\mathrm{PepT}_{\mathrm{So} 2}$, in which the two asparagine residues in $\mathrm{H} 12$ and the unpreserved ECL play key roles. Although the physiological significance of tetramer formation remains unknown, tetramer formation may contribute to stable expression and/or membrane localization. In all of the structures determined in this study, each $\mathrm{PepT}_{\text {So2 }}$ protomer adopts a similar inward-open state, which might reflect cooperative substrate transport in the tetramer. It is also possible that the oligomerization state of $\mathrm{PepT}_{\mathrm{So} 2}$ under physiological conditions is regulated by lipid molecules in the membrane, an unknown partner protein or the binding of substrates or additional cofactors. However, the molecular basis and the physiological significance of the oligomerization of membrane transporters, including $\mathrm{PepT}_{\mathrm{So} 2}$, remain unclear at many points and further research is awaited.

\section{Data availability}

Data supporting the findings of this manuscript are available from the corresponding authors upon reasonable request. The coordinates and structure factors from the X-ray crystallographic analyses have been deposited in the Protein Data Bank (PDB) with accession codes 6jkd and 6jkc for the form $A$ (space group I4) and form $B$ (space group $P 42_{1} 2$ ) crystals, respectively. X-ray diffraction images are also available from the Zenodo data repository (https://zenodo.org/record/ 2533841). The coordinates used for electron-microscopic analysis have been deposited in the PDB with accession code 6ji1. The EM map of $\mathrm{PepT}_{\mathrm{So} 2}$ embedded in Salipro nanoparticles has been deposited in the Electron Microscopy Data Bank (EMDB) under accession code EMD-9832.

\section{Acknowledgements}

We thank T. Nishizawa and T. Kusakizako for useful suggestions and discussions, and the beamline staff members (K. Hirata, N. Kawano and K. Yamashita) at BL32XU at SPring-8 for technical support during data collection. We also thank K. Iwasaki for help at the IPR cryo-EM facility. The $\mathrm{X}$-ray diffraction experiments were performed on BL32XU at SPring-8 (proposal No. 2017B2578) with the approval of RIKEN.

\section{Funding information}

The following funding is acknowledged: Japan Society for the Promotion of Science (grant No. 16H06294). This work was partially supported by the Platform Project for Supporting Drug Discovery and Life Science Research (Basis for Supporting Innovative Drug Discovery and Life Science Research; BINDS) from AMED under grant Nos. JP18am0101072 and JP18am0101075, by a Grant-in-Aid for Scientific Research (S) (24227004) and a Grant-in-Aid for Scientific Research (B) (25291011) from the Japan Society for the Promotion of Science (JSPS) to ON and RI, respectively, and by a Grant-in-Aid for JSPS Fellows.

\section{References}

Adams, P. D., Afonine, P. V., Bunkóczi, G., Chen, V. B., Davis, I. W., Echols, N., Headd, J. J., Hung, L.-W., Kapral, G. J., GrosseKunstleve, R. W., McCoy, A. J., Moriarty, N. W., Oeffner, R., Read, R. J., Richardson, D. C., Richardson, J. S., Terwilliger, T. C. \& Zwart, P. H. (2010). Acta Cryst. D66, 213-221.

Alguel, Y., Cameron, A. D., Diallinas, G. \& Byrne, B. (2016). Biochem. Soc. Trans. 44, 1737-1744.

Backmark, A. E., Olivier, N., Snijder, A., Gordon, E., Dekker, N. \& Ferguson, A. D. (2013). Protein Sci. 22, 1124-1132.

Caffrey, M. \& Cherezov, V. (2009). Nature Protoc. 4, 706-731.

Chen, Y.-J., Pornillos, O., Lieu, S., Ma, C., Chen, A. P. \& Chang, G. (2007). Proc. Natl Acad. Sci. USA, 104, 18999-19004.

Doki, S., Kato, H. E., Solcan, N., Iwaki, M., Koyama, M., Hattori, M., Iwase, N., Tsukazaki, T., Sugita, Y., Kandori, H., Newstead, S., Ishitani, R. \& Nureki, O. (2013). Proc. Natl Acad. Sci. USA, 110, 11343-11348.

Emsley, P. \& Cowtan, K. (2004). Acta Cryst. D60, 2126-2132.

Emsley, P., Lohkamp, B., Scott, W. G. \& Cowtan, K. (2010). Acta Cryst. D66, 486-501.

Frauenfeld, J., Löving, R., Armache, J.-P., Sonnen, A. F.-P., Guettou, F., Moberg, P., Zhu, L., Jegerschöld, C., Flayhan, A., Briggs, J. A. G., Garoff, H., Löw, C., Cheng, Y. \& Nordlund, P. (2016). Nature Methods, 13, 345-351.

Guettou, F., Quistgaard, E. M., Raba, M., Moberg, P., Löw, C. \& Nordlund, P. (2014). Nature Struct. Mol. Biol. 21, 728-731.

Guettou, F., Quistgaard, E. M., Trésaugues, L., Moberg, P., Jegerschöld, C., Zhu, L., Jong, A. J. O., Nordlund, P. \& Löw, C. (2013). EMBO Rep. 14, 804-810.

Hirata, K., Kawano, Y., Ueno, G., Hashimoto, K., Murakami, H., Hasegawa, K., Hikima, T., Kumasaka, T. \& Yamamoto, M. (2013). J. Phys. Conf. Ser. 425, 012002.

Kabsch, W. (2010). Acta Cryst. D66, 125-132.

Kawate, T. \& Gouaux, E. (2006). Structure, 14, 673-681.

Locher, K. P. (2016). Nature Struct. Mol. Biol. 23, 487-493.

Löw, C., Moberg, P., Quistgaard, E. M., Hedrén, M., Guettou, F., Frauenfeld, J., Haneskog, L. \& Nordlund, P. (2013). Biochim. Biophys. Acta, 1830, 3497-3508.

McCoy, A. J., Grosse-Kunstleve, R. W., Adams, P. D., Winn, M. D., Storoni, L. C. \& Read, R. J. (2007). J. Appl. Cryst. 40, 658-674.

Murshudov, G. N., Skubák, P., Lebedev, A. A., Pannu, N. S., Steiner, R. A., Nicholls, R. A., Winn, M. D., Long, F. \& Vagin, A. A. (2011). Acta Cryst. D67, 355-367.

Newstead, S., Drew, D., Cameron, A. D., Postis, V. L., Xia, X., Fowler, P. W., Ingram, J. C., Carpenter, E. P., Sansom, M. S., McPherson, M. J., Baldwin, S. A. \& Iwata, S. (2011). EMBO J. 30, 417-426.

Nishizawa, T., Kita, S., Maturana, A. D., Furuya, N., Hirata, K., Kasuya, G., Ogasawara, S., Dohmae, N., Iwamoto, T., Ishitani, R. \& Nureki, O. (2013). Science, 341, 168-172.

Parker, J. L., Li, C., Brinth, A., Wang, Z., Vogeley, L., Solcan, N., Ledderboge-Vucinic, G., Swanson, J. M. J., Caffrey, M., Voth, G. A. \& Newstead, S. (2017). Proc. Natl Acad. Sci. USA, 114, 1318213187.

Pettersen, E. F., Goddard, T. D., Huang, C. C., Couch, G. S., Greenblatt, D. M., Meng, E. C. \& Ferrin, T. E. (2004). J. Comput. Chem. 25, 1605-1612.

Potterton, L., Agirre, J., Ballard, C., Cowtan, K., Dodson, E., Evans, P. R., Jenkins, H. T., Keegan, R., Krissinel, E., Stevenson, K., Lebedev, A., McNicholas, S. J., Nicholls, R. A., Noble, M., Pannu, N. S., Roth, C., Sheldrick, G., Skubak, P., Turkenburg, J., Uski, V., von Delft, F., Waterman, D., Wilson, K., Winn, M. \& Wojdyr, M. (2018). Acta Cryst. D74, 68-84.

Punjani, A., Rubinstein, J. L., Fleet, D. J. \& Brubaker, M. A. (2017). Nature Methods, 14, 290-296. 
Reyes, N., Ginter, C. \& Boudker, O. (2009). Nature (London), 462, 880-885.

Scheres, S. H. W. \& Chen, S. (2012). Nature Methods, 9, 853-854.

Tabata, S., Kitago, Y., Fujii, Y., Mihara, E., Tamura-Kawakami, K., Norioka, N., Takahashi, K., Kaneko, M. K., Kato, Y. \& Takagi, J. (2018). Protein Expr. Purif. 147, 94-99.

Tan, Y. Z., Baldwin, P. R., Davis, J. H., Williamson, J. R., Potter, C. S., Carragher, B. \& Lyumkis, D. (2017). Nature Methods, 14, 793-796.

Yamashita, K., Hirata, K. \& Yamamoto, M. (2018). Acta Cryst. D74, 441-449.
Yernool, D., Boudker, O., Jin, Y. \& Gouaux, E. (2004). Nature (London), 431, 811-818.

Zhang, K. (2016). J. Struct. Biol. 193, 1-12.

Zhao, Y., Mao, G., Liu, M., Zhang, L., Wang, X. \& Zhang, X. C. (2014). Structure, 22, 1152-1160.

Zheng, S. Q., Palovcak, E., Armache, J., Verba, K. A., Cheng, Y. \& Agard, D. A. (2017). Nature Methods, 14, 331-332.

Zivanov, J., Nakane, T., Forsberg, B. O., Kimanius, D., Hagen, W. J. H., Lindahl, E. \& Scheres, S. H. W. (2018). Elife, 7, e42166. 